\title{
Genome-wide gene expression profiling identifies overlap with malignant adrenocortical tumours and novel mechanisms of inefficient steroidogenesis in familial ACTH-independent macronodular adrenal hyperplasia
}

\section{Dear Editor}

ACTH-independent macronodular adrenal hyperplasia (AIMAH) is a rare cause of late-onset sporadic Cushing's syndrome (CS; Lacroix 2009). Discordance between massive adrenal hyperplasia and relatively mild hypercortisolism is distinctive of AIMAH, ascribed to differential localisation of steroidogenic enzymes in clear and compact cells, resulting in inefficient steroidogenesis (Lacroix 2009). Thus, CS is the result of hypercortisolism due to a colossal increase in adrenocortical cell mass. Iodocholesterol scintigraphy typically shows bilateral uptake consistent with bilateral adrenal hyperfunction, although unilateral or asymmetrical uptake may occur, guiding adrenal selection for resection (Gagliardi et al. 2009). We and others have reported AIMAH kindreds providing compelling evidence for an inherited basis in some cases (Gagliardi et al. 2009). The genetic basis of familial AIMAH is unknown, although segregation analysis suggests autosomal dominant inheritance (Gagliardi et al. 2009).

Cortisol secretion in AIMAH tumours may be regulated by aberrantly expressed (non-ACTH) G-protein-coupled receptors (GPCR; e.g. vasopressin (VP); Lacroix 2009). Whilst the biological basis for aberrant GPCR expression is unknown, in a murine model, it sufficed for the development of a phenotype reminiscent of human AIMAH (Mazzuco et al. 2006). This suggests that aberrant GPCR expression occurs early if it is not primary in the pathogenesis of AIMAH; alternatively, it could represent an epiphenomenon of tumorigenesis.

The pathogenesis of AIMAH, a cytologically benign disease without propensity for metastasis, is unknown. Molecular studies of sporadic AIMAH suggest altered WNT and protein kinase A signalling and microRNAs in its pathogenesis (Bimpaki et al. 2010, Almeida et al. 2011).
To our knowledge, transcriptome profiling of familial AIMAH has not been performed.

We have previously reported an AIMAH kindred (AIMAH-01), the proband of which, aged 69 years, presented with florid CS (Gagliardi et al. 2009). Bilateral adrenalectomy (combined weight $130 \mathrm{~g}$ ) was curative, but he died from recurrent sepsis. His siblings, aged 61 and 65 years, presented with early CS; whilst lacking a Cushingoid phenotype, they had hypertension, mood change and central obesity. Iodocholesterol scintigraphy guided adrenalectomy of the dominant gland (weights 55 and $50 \mathrm{~g}$ respectively). One sibling became eucortisolaemic and the other transiently hypocortisolaemic - hypertension improved in both. Limited aberrant receptor testing revealed that all had an aberrant cortisol response to VP (Gagliardi et al. 2009). RT quantitative PCR (RT-qPCR) demonstrated that the tumours overexpressed the VP receptor 1A (AVPR1A) and ectopically expressed AVPRIB (Gagliardi et al. 2009).

Using Affymetrix Human GeneChip HumanGene 1.0 ST arrays, we compared the transcriptomes of the three available familial AIMAH-01 tumours with two normal adrenal glands and analysed the data for differential expression and using Ingenuity Pathway Analysis, Gene Set Enrichment Analysis (GSEA) and Motif Activity Response Analysis (MARA). We performed RT-qPCR validation of selected differentially expressed genes (DEG). Our aim was to determine the molecular mechanisms involved in the pathogenesis of familial AIMAH and the inefficient steroidogenesis which epitomises the tumours.

There were 367 significant DEG (162 upregulated). Global gene expression differences between AIMAH-01 and the normal samples were greater than within-group differences in AIMAH-01 (Fig. 1). The 50 most upand downregulated significant DEG are shown in the 


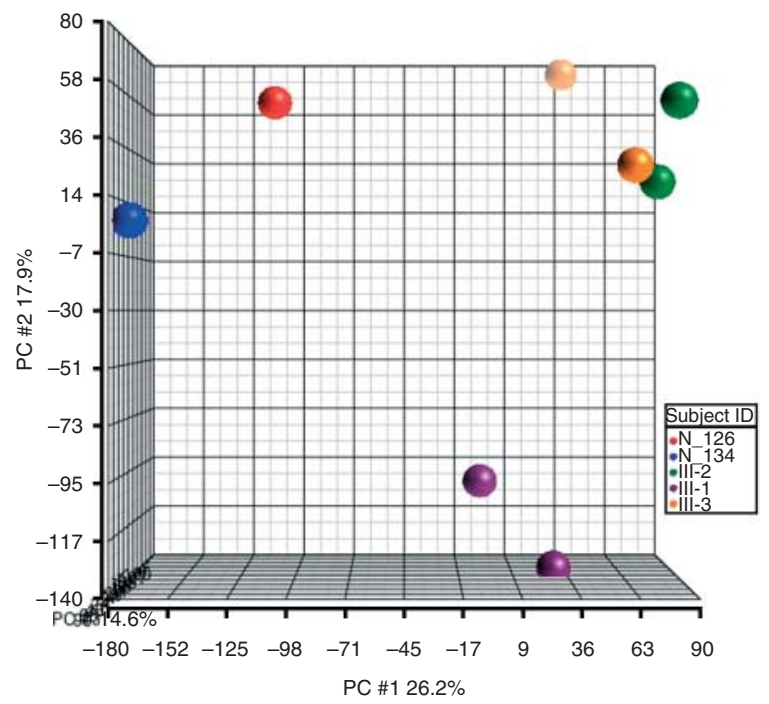

Figure 1 Principal component analysis plot of AIMAH-01 (III-1, III-2 and III-3) tumours and normal adrenal cortex (N_126 and N_134). The first principal component (PC \#1) is shown on the $x$-axis and explains the maximum amount $(26.2 \%)$ of variation in the gene expression data. The second principal component (PC \#2) is shown on the $y$-axis and explains the second most amount of remaining variation in the gene expression data (17.9\%). III-1, proband; III-2 and III-3, siblings. This figure was generated using Partek Genomics Suite, version 6.4 (Partek Incorporated, St Louis, MO, USA).

heat map (Fig. 2); we validated, by RT-qPCR, seven of the ten most DEG. The ACTH receptor was not differentially expressed. The glucocorticoid receptor was downregulated (2.5-fold; $P=0.056$ ).

Microarray analysis suggested reduced expression of the cholesterol side-chain cleavage enzyme (CYP11A1). Whilst not statistically significant by RT-qPCR (sevenfold, $P=0.09$ ), the downregulation may be biologically important in contributing to inefficient steroidogenesis because CYP11A1 regulates the rate-limiting conversion of cholesterol to pregnenolone (Miller 2008). Furthermore, AIMAH is clinically and genetically heterogeneous; hence, more severe synthetic defects may be present in other cells (Lacroix 2009). Aldosterone synthase was downregulated (13-fold; $P=0.004$ ). The other steroidogenic enzymes studied were not differentially expressed.

Transcription factor activity profiling (MARA) predicted reduced activities of $\mathrm{Sp} 1$ transcription factor (SP1) and steroidogenic factor-1 (NR5A1), the transcriptional regulators of the rate-limiting enzymes of steroidogenesis, CYP11A1 and steroidogenic acute regulatory protein (Miller 2008). However, SP1 and NR5A1 were not DEG. We postulate that reduced activity may be due to altered post-translational modification of their proteins (e.g. lowering activity, increasing degradation or altering interactions with coactivators). Downregulation of steroidogenic enzymes due to reduced transcription factor activity is a novel mechanism of inefficient steroidogenesis in AIMAH.

Most highly over-represented amongst the DEG was the 'Cellular effects of Sildenafil' signalling pathway. Sildenafil's cellular target, PDE5A, was not a DEG. The microarray data suggested downregulation of $P D E 1 C$ and PDE2A; these were not statistically significant by RT-qPCR. Nevertheless, the downregulation could be biologically significant, increasing intracellular cAMP signalling. These data suggest that dysregulated cAMP signalling, already implicated in adrenocortical tumorigenesis, may also be involved in the pathogenesis of AIMAH-01 tumours (Giordano et al. 2009).

The signalling pathways regulated by cardiac $\beta$-adrenergic and dopamine receptor signalling were over-represented. It is not known whether these GPCR are coupled to steroidogenesis in AIMAH-01. There was no cortisol response to $\beta$-adrenergic stimulation in the only patient available for such evaluation. Evaluation for an aberrant response to dopamine agonists is not part of the recommended screening protocol and was not examined (Lacroix 2009). We found discordant enrichment for genes regulated by ACTH in murine Y1 adrenal cells, consistent with the prevailing concept of the ACTH independence of tumorigenesis in AIMAH (Antonini et al. 2006).

DEG functions included cellular growth, proliferation, signalling and interaction. Some of the most highly DEG in our dataset have known roles in tumorigenesis and metastasis (e.g. myosin heavy chain 11, smooth muscle, MYH11; matrix metallopeptidase 16 (membrane inserted), MMP16; hyaluronan synthase 2,HAS2); some of these were also recently reported as DEG in sporadic AIMAH (Almeida et al. 2011). MARA suggested that upregulation of MMP16; HAS2 may be due to increased activity of their transcriptional regulators.

We found enrichment for DEG in adrenocortical carcinoma (ACC) compared with normal adrenal gland/cortex or adrenocortical adenoma and for ACC with an aggressive, malignant clinical course (Giordano et al. 2009). Overall, most of the DEG and the GSEA data, the latter derived from integrated comparisons of our transcriptome data with previously published DEG sets, suggest that DEG are shared between AIMAH and malignant adrenocortical tumours. Our findings are substantiated by data from other studies. Several differentially expressed 


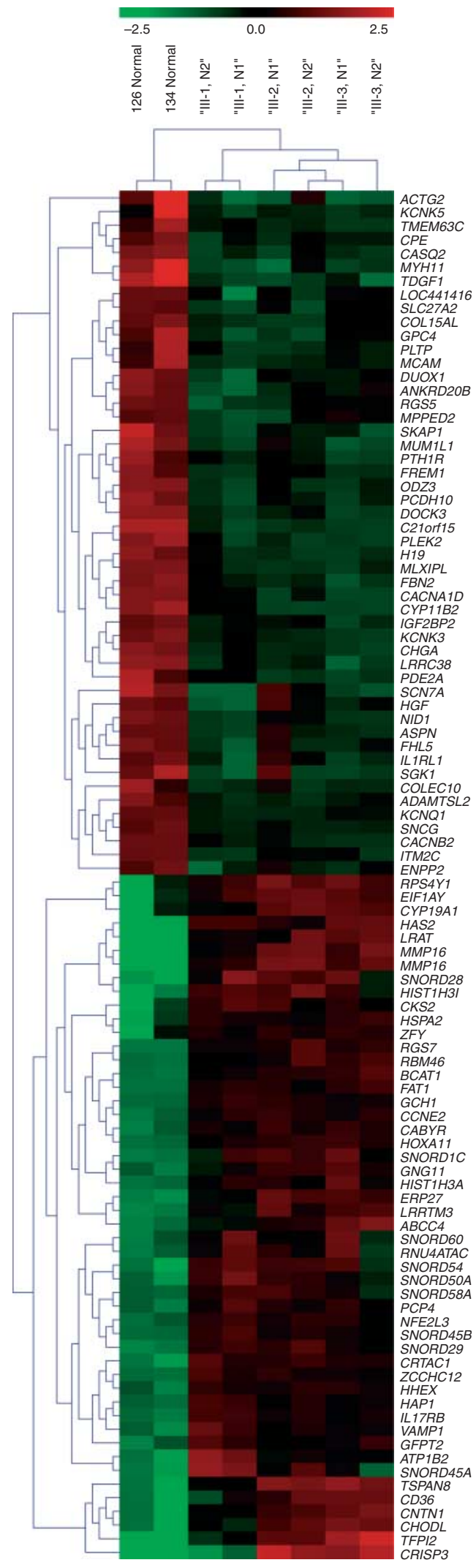

microRNAs in sporadic AIMAH were previously implicated in tumorigenesis or metastasis (Bimpaki et al. 2010). In another study, gene expression profiling of several AIMAH nodules from one patient identified aberrant expression of oncogenic pathways in larger nodules (Almeida et al. 2011). These observations are intriguing given the benign biology of AIMAH, without propensity for metastasis. We therefore surmise that other genetic changes in target or supporting cells are not conducive to malignancy.

AIMAH tumours are regarded benign as metastases have never been documented clinically in the longterm post-operative surveillance of patients (Lacroix 2009). Whether subclinical metastases occur is unknown, as radiological surveillance is not performed. If AIMAH cells did have metastatic potential, then due to inefficient steroidogenesis, which in a metastatic cell might be even more pronounced, several decades might be required before manifesting clinically with CS. This may never eventuate, due to the late initial age of onset of AIMAH.

We found enrichment for DEG in sporadic AIMAH with mixed or no aberrant cortisol responses, suggesting that DEG are not specific to the aberrant receptor(s) being expressed and that molecular mechanisms may be shared between sporadic and familial AIMAH tumours - a novel concept as regards AIMAH. We did not identify mechanisms underlying VP receptor overexpression. However, glucocorticoids may upregulate $A V P R 1 A$, and VP may be involved in the paracrine regulation of cortisol secretion (Perraudin et al. 1993). Thus, eutopic overexpression of AVPRIA in AIMAH-01 may be secondary to tumorigenesis and hypercortisolism; the augmented in vivo cortisol response to VP may reflect the normal coupling of (overexpressed) AVPR1A to steroidogenesis. The mechanism of ectopic AVPRIB expression has not been elucidated.

The limitations of this study are the small numbers of samples evaluated and that we performed a 'whole tissue' gene expression study. AIMAH exhibits cellular heterogeneity and there are likely to be DEG

Figure 2 Heat map of 50 most upregulated (according to fold change) and 50 most downregulated genes in the AIMAH-01 tumours compared with normal adrenal cortex. The expression values of the probe sets were adjusted so that each probe set had the same mean expression value. Red indicates expression above the mean expression value for all probe sets; green indicates expression below the mean expression value for all probe sets. Normal: N126 and N134; AIMAH-01: III-1, III-2 and III-3; N1, 2: nodule 1, 2. This figure was generated in Multiexperiment Viewer program. 
between cell types (e.g. steroidogenic enzymes in clear and compact cells) that may be diluted by studying the whole tissue, without having performed cellular partitioning. Partial characterisation of aberrant receptor expression by us and others in AIMAH is another limitation. However, complete characterisation of aberrant GPCR expression is not possible given that novel GPCR continue to be identified (Assie et al. 2010).

Ours is the first study to profile the transcriptome of familial AIMAH tumours. Using novel analytical approaches, we have identified involvement of pathways in familial AIMAH that have been implicated in sporadic AIMAH or other adrenocortical tumours, suggesting that mechanisms of tumorigenesis are shared between familial AIMAH and other sporadic adrenocortical tumours. This is a novel concept. We also found novel mechanisms of inefficient steroidogenesis in the familial AIMAH tumours. Our data are based on a small number of tumours studied, thus additional transcriptome studies of familial AIMAH are required. Ultimately, the identification of the genetic basis of familial AIMAH should enable further progress towards understanding the pathogenesis of these hereditary adrenocortical tumours.

$$
\begin{array}{r}
\text { Lucia Gagliardi }^{1,2} \\
\text { King-Hwa Ling } \\
\text { Chung H Kok }^{4} \\
\text { Joseph Carolan }^{5} \\
\text { Peter Brautigan } \\
\text { Rosalie Kenyon } \\
6,7 \\
\text { Richard J D'Andrea }{ }^{2,4,7,8,9} \\
\text { Mark Van der Hoek } \\
\text { Christopher N Hahn } \\
\text { 2,5 } \\
\text { David J Torpy }^{1,2} \\
\text { Hamish S Scott }^{2,5,7}
\end{array}
$$

${ }^{1}$ Endocrine and Metabolic Unit, Royal Adelaide Hospital, Adelaide, South Australia 5000, Australia ${ }^{2}$ School of Medicine, University of Adelaide, Adelaide, South Australia 5000, Australia

${ }^{3}$ Department of Obstetrics and Gynaecology, Faculty of Medicine and Health Sciences, Universiti Putra Malaysia, 43400 UPM Serdang, Selangor, Malaysia Departments of ${ }^{4}$ Haematology, ${ }^{5}$ Molecular Pathology, and ${ }^{6}$ Adelaide Microarray Centre, Centre for Cancer

Biology, SA Pathology, Frome Road, Adelaide, South Australia 5000, Australia

${ }^{7}$ School of Molecular and Biomedical Science, University of Adelaide, Adelaide, South Australia 5000, Australia
${ }^{8}$ Centre for Stem Cell Research, University of Adelaide, Adelaide, South Australia 5000,

Australia

${ }^{9}$ Haematology and Oncology Department, The Queen Elizabeth Hospital, Woodville, South Australia 5011,

Australia

(Correspondence should be addressed to L Gagliardi who is now at Department of Genetics and Molecular Pathology, SA Pathology, Ground Floor, Frome Road, Adelaide SA 5000, South Australia, Australia; Email: lucia.gagliardi@health.sa.gov.au.)

\section{Declaration of interest}

L Gagliardi, C N Hahn, D J Torpy and H S Scott are currently in receipt of a Royal Adelaide Hospital Clinical Project Grant (2011) for continued related research work. The other authors have no declarations.

\section{Funding}

This work was supported by a Royal Adelaide Hospital/Institute of Medical and Veterinary Science Clinical Project Grant. This work was supported by an Endocrine Society of Australia Higher Research Degree Scholarship (January 2008 to August 2008) and a National Health and Medical Research Council (NHMRC) Medical Postgraduate Research Scholarship (August 2008 to March 2011; L Gagliardi) and a NHMRC fellowship 461204 (H S Scott).

\section{Acknowledgements}

We sincerely thank Prof. Michael Stowasser and Associate Prof. David Nicol, Princess Alexandra Hospital, Brisbane, for providing the normal adrenal samples and Dr Mikhail Pachkov, Swiss Institute of Bioinformatics, for performing Motif Activity Response Analysis.

\section{References}

Almeida MQ, Harran M, Bimpaki EI, Hsiao HP, Horvath A, Cheadle C, Watkins T, Nesterova M \& Stratakis CA 2011 Integrated genomic analysis of nodular tissue in macronodular adrenocortical hyperplasia: progression of tumourigenesis in a disorder associated with multiple benign lesions. Journal of Clinical Endocrinology and Metabolism 96 E278-E238. (doi:10.1210/jc.2010-2420)

Antonini SR, Baldacchino V, Tremblay J, Hamet P \& Lacroix A 2006 Expression of ACTH receptor pathway genes in glucose-dependent insulinotrophic peptide (GIP)-dependent Cushing's syndrome. Clinical Endocrinology 64 29-36. (doi:10.1111/j.1365-2265. 2005.02411.x)

Assie G, Louiset E, Sturm N, René-Corail F, Groussin L, Bertherat J, Thomas M, Lefebvre H, Feige JJ, Clauser E 
et al. 2010 Systematic analysis of $\mathrm{G}$ protein-coupled receptor gene expression in adrenocorticotropinindependent macronodular adrenocortical hyperplasia identifies novel targets for pharmacological control of adrenal Cushing's syndrome. Journal of Clinical Endocrinology and Metabolism 95 E253-E262. (doi:10.1210/jc.2009-2281)

Bimpaki EI, Iliopoulos D, Moraitis A \& Stratakis CA 2010 MicroRNA signature in massive macronodular adrenocortical disease and implications for adrenocortical tumourigenesis. Clinical Endocrinology $\mathbf{7 2}$ 744-751. (doi:10.1111/j.1365-2265.2009.03725.x) Gagliardi L, Hotu C, Casey G, Braund WJ, Ling KH, Dodd T, Manavis J, Devitt PG, Cutfield R, Rudzki Z et al. 2009 Familial vasopressin-sensitive ACTHindependent macronodular adrenal hyperplasia (VPs-AIMAH): clinical studies of three kindreds. Clinical Endocrinology 70 883-891. (doi:10.1111/j. 1365-2265.2008.03471.x)

Giordano TJ, Kuick R, Else T, Gauger PG, Vinco M, Bauersfeld J, Sanders D, Thomas DG, Doherty G \& Hammer G 2009 Molecular classification and prognostication of adrenocortical tumours by transcriptome profiling. Clinical Cancer Research 15 668-676. (doi:10.1158/1078-0432.CCR-08-1067)

Lacroix A 2009 ACTH-independent macronodular adrenal hyperplasia. Best Practice \& Research. Clinical Endocrinology \& Metabolism 23 245-259. (doi:10.1016/ j.beem.2008.10.011)

Mazzuco TL, Chabre O, Feige JJ \& Thomas M 2006 Aberrant expression of human luteinising hormone receptor by adrenocortical cells is sufficient to provoke both hyperplasia and Cushing's syndrome features. Journal of Clinical Endocrinology and Metabolism 91 196-203. (doi:10.1210/jc.2005-1975)

Miller WL 2008 Steroidogenic enzymes. In Disorders of the Human Adrenal Cortex. Endocr Dev, pp 1-18. Eds CE Flück \& WL Miller. Basel: Karger. (doi:10.1159/000134751)

Perraudin V, Delarue C, Lefebvre H, Contesse V, Kuhn JM \& Vaudry H 1993 Vasopressin stimulates cortisol secretion from human adrenocortical tissue through activation of V1 receptors. Journal of Clinical Endocrinology and Metabolism 76 1522-1528. (doi:10.1210/ jc.76.6.1522) 